\title{
A Novel Model of Atherosclerosis in Rabbits Using Injury to Arterial Walls Induced by Ferric Chloride as Evaluated by Optical Coherence Tomography as well as Intravascular Ultrasound and Histology
}

\author{
Jinwei Tian, ${ }^{1}$ Sining Hu, ${ }^{1}$ Yanli Sun, ${ }^{1}$ Xiang Ban, ${ }^{2}$ Huai Yu, ${ }^{1}$ Nana Dong, ${ }^{1}$ Jian Wu, ${ }^{1}$ and Bo Yu ${ }^{1}$ \\ ${ }^{1}$ Department of Cardiology, Key Laboratories of Education, Ministry for Myocardial Ischemia Mechanism and Treatment, \\ 2nd Affiliated Hospital of Harbin Medical University, Harbin 150086, China \\ ${ }^{2}$ Department of Pathology, Harbin Medical University, Harbin 150086, China
}

Correspondence should be addressed to Bo Yu, yubodr2009@163.com

Received 16 January 2012; Revised 6 March 2012; Accepted 10 March 2012

Academic Editor: Michael Kalafatis

Copyright (C) 2012 Jinwei Tian et al. This is an open access article distributed under the Creative Commons Attribution License, which permits unrestricted use, distribution, and reproduction in any medium, provided the original work is properly cited.

\begin{abstract}
This study aim was to develop a new model of atherosclerosis by $\mathrm{FeCl}_{3}$-induced injury to right common carotid arteries (CCAs) of rabbits. Right CCAs were induced in male New Zealand White rabbits $(n=15)$ by combination of a cholesterol-rich diet and $\mathrm{FeCl}_{3}$-induced injury to arterial walls. The right and left CCAs were evaluated by histology and in vivo intravascular ultrasound (IVUS) and optical coherence tomography (OCT) examinations of 24 hours $(n=3)$, 8 weeks $(n=6)$, and 12 weeks $(n=6)$ after injury. Each right CCA of the rabbits showed extensive white-yellow plaques. At eight and 12 weeks after injury, IVUS, OCT, and histological findings demonstrated that the right CCAs had evident eccentric plaques. Six plaques (50\%) with evident positive remodeling were observed. Marked progression was clearly observed in the same plaque at 12 weeks after injury when it underwent repeat OCT and IVUS. We demonstrated, for the first time, a novel model of atherosclerosis induced by $\mathrm{FeCl}_{3}$. The model is simple, fast, inexpensive, and reproducible and has a high success rate. The eccentric plaques and remodeling of plaques were common in this model. We successfully carried out IVUS and OCT examinations twice in the same lesion within a relatively long period of time.
\end{abstract}

\section{Introduction}

Atherosclerosis is one of the leading causes of acute coronary syndrome and ischemic stroke. The use of cell culture lacks the complexity of real models of disease. Hence, the use of laboratory animals is considered to be necessary to study the mechanism of atherosclerosis and to evaluate newer antiatherosclerotic strategies [1]. Several animal models of atherosclerosis have been reported for better understanding of atherosclerosis [1]. However, there is no "gold standard" animal model for atherosclerosis. Some authors using animal models have used ferric chloride $\left(\mathrm{FeCl}_{3}\right)$ to induce oxidative injury to study the mechanisms and therapeutic strategies of neointima proliferation and thrombus formation $[2,3]$. It is not clear if a combination of $\mathrm{FeCl}_{3}$-induced injury to the artery wall and a high-cholesterol diet can promote atherosclerosis in the common carotid artery (CCA) of rabbits.

Intravascular ultrasound (IVUS) offers good penetration of tissue $(10 \mathrm{~mm})$ and is used extensively to accurately quantify the volume of atherosclerotic plaques [4]. It also monitors dynamically the progression of atherosclerosis as well as the effects of antiatherosclerotic strategies. Optical coherence tomography (OCT) has high resolution $(\leq 10$ $20 \mu \mathrm{m}$ ) [4]. It has emerged as one of the most promising tools to assess acute coronary syndrome and to detect key features of plaques at high risk of rupture. This is due to its ability to differentiate plaque composition [5], such as macrophage infiltration and lipid content. Therefore, a combination of IVUS and OCT can complement each other, obtain comprehensive information about arterial wall, and evaluate the features of the model [4]. 
The aims of the present study were to (i) develop a new model of atherosclerosis using rabbits as evaluated by histology and in vivo IVUS and OCT examinations and (ii) investigate the feasibility of IVUS and OCT in a serial study of the development of atherosclerotic lesions in this model.

\section{Materials and Methods}

2.1. Ethical Approval of the Study Protocol. The present study was undertaken after approval from the Scientific Affairs Committee on Animal Research and Ethics of the Second Affiliated Hospital of Harbin Medical University. All procedures using animals were conducted in compliance with the administrative acts for experimental animals in China.

2.2. Experimental Design. Carotid atherosclerosis was induced in male New Zealand White rabbits $(n=15)$ by combination of a cholesterol-rich diet (containing 1.5\% cholesterol) and $\mathrm{FeCl}_{3}$-induced injury to arterial walls. One week after commencing a cholesterol-rich diet, all rabbits were anesthetized (all via the intraperitoneal route) with ketamine $(35 \mathrm{mg} / \mathrm{kg})$, xylazine $(5 \mathrm{mg} / \mathrm{kg})$, and acepromazine $(0.75 \mathrm{mg} / \mathrm{kg})$. Anesthesia was maintained during the procedure with isoflurane inhalation via a facemask. Rabbits were then placed in dorsal recumbency. A midline neck incision was made, and right CCA was surgically exposed. $\mathrm{FeCl}_{3}$ induced injury to the arterial wall was carried out similarly to that carried out in mice as described other studies $[2,6]$. A preliminary study was done to establish the highest concentration of $\mathrm{FeCl}_{3}$ needed and the time needed for injury. The right CCA was carefully dissected and then injured by placing a strip of filter paper $(0.5 \times 2.0 \mathrm{~mm})$ saturated in $5 \% \mathrm{FeCl}_{3}$ solution onto the adventitia of the midportion of the artery for 5 minutes. After removal of the filter paper, the incision was sutured closed. Carotid blood flow was monitored before and after injury using ultrasound. Twenty-four hours after injury, 3 rabbits were randomly selected, and OCT was carried out. At 8 weeks, 6 rabbits from the remaining 12 rabbits underwent OCT and IVUS at both CCAs. They were then killed for histological analyses of tissue. The remaining 6 rabbits underwent OCT and IVUS at week 8 and repeat imaging at week 12. Images from OCT and IVUS from different time points were coregistered using the origin of the CCA as an anatomic landmark.

2.3. OCT Imaging and Analyses. After anesthesia as described above, OCT of the right CCA was done before killing the rabbits. The OCT system used in the present study has been described previously [7]. Briefly, a 0.016-inch OCT catheter (ImageWire; LightLab Imaging, Westford, MA, USA) was advanced to the origin of the CCA through a 3-F occlusion balloon catheter, through which $200 \mu \mathrm{g}$ of nitroglycerin were given. Serial OCT images were obtained in an automated pullback format at $1 \mathrm{~mm} / \mathrm{s}$ and 15 frames/s during continuous saline flush at $3 \mathrm{~mL} / \mathrm{s}$ a through the guiding catheter to transiently displace blood. All OCT images were analyzed offline by two reviewers blinded to the protocol. As described previously $[8,9]$, the OCT features of atherosclerotic lesions were defined as loss of the normal "layered" appearance of the vessel wall with (i) an increase in the intimal area, (ii) the presence of highly reflective subintimal areas, and (iii) discontinuation of the internal elastic membrane. The range of OCT images with atherosclerosis was semiquantified according to the maximal value of the involved arc on crosssections.

2.4. IVUS Imaging and Analyses. IVUS was carried out after administration of $200 \mu \mathrm{g}$ of nitroglycerin using a $40-\mathrm{MHz}$ catheter (Atlantis SR Pro; Boston Scientific, Natick, MA, USA) and an automatic pullback device at $0.5 \mathrm{~mm} / \mathrm{s}$. All IVUS images were obtained at 30 frames/s and recorded on high-resolution super VHS videotapes. These were subsequently analyzed offline by two reviewers blinded to the protocol using manual planimetry software to trace the leading edges of the borders of the lumen and external elastic membrane. Every 30th image was analyzed, generating a series of cross-sections spaced exactly $0.5 \mathrm{~mm}$ apart. Plaque area was calculated as the cross-sectional area of the external elastic membrane (EEM) minus the luminal cross-sectional area. The maximal area was selected to subsequent analysis. Remodeling index was defined by calculating the ratio of the EEM area at the site with atherosclerosis, compared with the reference EEM area calculated as the average of proximal and distal reference sites. Remodeling was categorized as positive (remodeling index $(\mathrm{RI})>1.05)$ or none $(\mathrm{RI}<1.05)$ at that site.

2.5. Histology and Immunohistochemistry. All rabbits were killed by an overdose of pentobarbital sodium (IV). CCAs were quickly removed and perfused in phosphate-buffered saline. They were fixed in 4\% paraformaldehyde fixative and embedded in paraffin for staining with hematoxylin and eosin (H\&E), Masson's trichrome, and immunohistochemistry. Serial $4-\mu \mathrm{m}$-thick cross-sections were stained with $\mathrm{H} \& \mathrm{E}$ stain. Collagen contents were also determined (indicated in blue color with Masson's trichrome staining). Immunostaining was done using antismooth muscle $\alpha$-actin antibody ( $1: 500$ dilutions; Sigma-Aldrich, Deisenhofen, Germany) to identify smooth muscle cells (SMCs) and anti-CD68 antibody (1:600 dilution; DAKO, Carpinteria, CA, USA) to identify macrophages. Immunohistochemical staining was visualized using a diaminobenzidine (DAB) kit according to manufacturer instructions. After immunostaining, tissue sections were counterstained with hematoxylin. All images were analyzed by Image-Pro Plus 4.5 (Media Cybernetics, Silver Spring, MD, USA). The collagen content, macrophages, and SMCs of atherosclerotic plaques were quantified and expressed as the percentage of plaque area.

2.6. Profile of Plasma Lipids. Plasma levels of total cholesterol (TC), triglyceride (TG), and low-density lipoprotein cholesterol (LDL-) C were determined by enzymatic assays of blood samples. These were collected from the ear veins of rabbits fasted overnight. Blood samples were centrifuged at $3000 \mathrm{~g}$ for $15 \mathrm{~min}$ at $4^{\circ} \mathrm{C}$ before the introduction of a highcholesterol diet and at 0 weeks, 8 weeks, and 12 weeks after 
surgery. Levels of high-density lipoprotein cholesterol (HDLC) were measured after precipitation of apolipoprotein Bcontaining lipoproteins using polyethylene glycol.

2.7. Statistical Analyses. All data analyses were undertaken using the SPSS program SPSS ver. 15.0 (SPSS, Chicago, IL, USA). Serum lipid levels, histological data, and IVUS parameters are presented by the mean \pm SD. The independent $t$-test was used to test for differences between two independent data. Comparisons between baseline and followup values in the same rabbit were made with the paired $t$-test. $P<0.05$ was considered significant.

\section{Results}

3.1. Serum Lipid Levels. At the eight weeks after injury, compared with baseline values, TC levels were raised by about 19fold (from $54 \pm 19$ to $1015 \pm 118 \mathrm{mg} / \mathrm{dL} ; P<0.001$ ), LDL-C levels were increased by about 20 -fold (from $32 \pm 12$ to $637 \pm$ $157 \mathrm{mg} / \mathrm{dL} ; P<0.001$ ), TG levels around sixfold (from $27 \pm 9$ to $150 \pm 53 \mathrm{mg} / \mathrm{dL} ; P=0.004$ ), and HDL-C levels around twofold ( $16 \pm 7$ to $32 \pm 13 \mathrm{mg} / \mathrm{dL} ; P=0.001$ ). Levels of TC, LDL-C, TG, and HDL-C did not show a significant change from 8 weeks after injury to 12 weeks after injury.

3.2. Histological and Immunohistochemical Findings. Extensive white-yellow plaques in right CCAs could be seen (Figure 1(a)). At eight weeks and 12 weeks after injury, the intimal surfaces of arteries in arterial sections from left CCAs were smooth and thin (Figure 1(b)). At eight weeks after injury, sections from right CCAs had eccentric plaques (Figure 1(c)). At twelve weeks after injury, the sections from right CCAs had more severe atherosclerosis (Figure $1(\mathrm{~d})$ ). The plaque area was significantly larger at 12 weeks after injury than at 8 weeks after injury $(0.82 \pm 0.16$ versus $0.34 \pm$ $\left.0.12 \mathrm{~mm}^{2} ; P<0.001\right)$.

Masson's trichrome analyses have shown that the collagen content was significantly higher at 8 weeks after injury than at 12 weeks after injury $(66.1 \pm 12.3 \%$ versus $47.2 \pm$ $10.2 \%, P=0.02)$. The plaques of this model were characterized by an obvious lipid core with an overlaying fibrous cap (Figures 2(b), 2(c), and 2(d)). No evident atherosclerosis was seen in the section from the left CCA (Figure 2(a)). The right CCAs of rabbits at 12 weeks after injury had more serious atherosclerosis (Figure 2(c)) than those at 8 weeks after injury (Figure 2(b)).

Immunohistochemical analyses showed that macrophage content was considerable at 8 weeks after injury and at 12 weeks after injury $(22.5 \pm 6.8 \%$ versus $25.0 \pm 6.0 \% ; P=0.53)$. Notably, the distribution of macrophages was mainly in the media 8 weeks after injury (Figure 3(a)), whereas it was in the intima 12 weeks after injury (Figure 3(b)). The content of SMCs was significantly higher at 12 weeks after injury than at 8 weeks after injury $(48.7 \pm 7.9 \%$ versus $32.7 \pm 10.1 \%$, $P=0.01)$. The SMCs were mainly distributed in the intima 8 weeks after injury (Figure 3(c)) and in the intima and media 12 weeks after injury (Figure 3(d)).

3.3. OCT Findings. At twenty-four hours after injury, no thrombus was detected in the vessel wall (Figure 4(a)). At

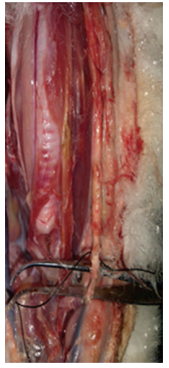

(a)

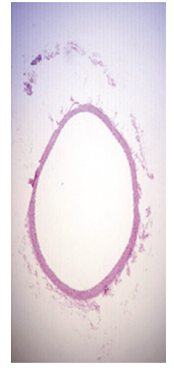

(b)

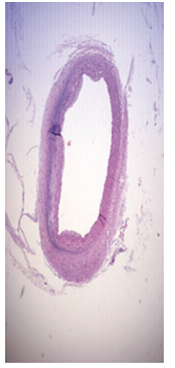

(c)

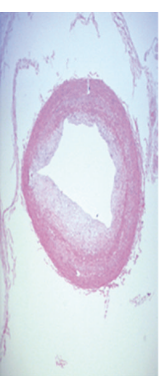

(d)
Figure 1: Atherosclerotic lesion (macroscopic en face view of the plaque) (a), H\&E staining of left common carotid arteries (b), and right common carotid arteries at 8 weeks (c) and 12 weeks (d) after injury. Original magnification, $\times 40$.

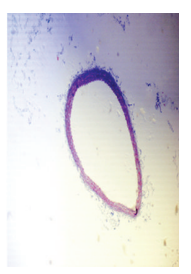

(a)

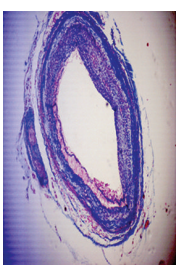

(b)

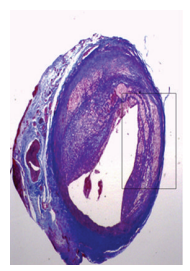

(c)

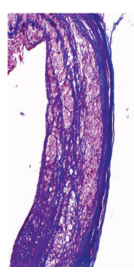

(d)
FIGURE 2: Masson's trichrome of the left common carotid arteries (a) and the right common carotid arteries at 8 weeks (b) and at 12 weeks ((c) and (d)) after injury. Panels (a) and (b) were derived from the same rabbit. Panel (d) was a higher magnification taken from the black box in Panel (c) and shows a forming fibrous cap over a lipid core. The collagen content was significantly higher at 8 weeks (b) after injury than at 12 weeks (c) after injury. The plaques of this model are characterized by an obvious lipid core with an overlaying fibrous cap ((b), (c), and (d)). No evident atherosclerosis was seen in the left common carotid artery (a). original magnification, $\times 40((a)-(c))$ and $\times 100(d)$.

eight weeks after injury, OCT images revealed atherosclerosis of right CCAs of all rabbits (Figure 4(b)), whereas atherosclerosis was not observed in left CCAs (Figure 4(c)). At twelve weeks after injury, progression of atherosclerosis of right CCAs was evident in the 6 rabbits that underwent repeat OCT (Figure 4(d)). The range of cross-sectional OCT images with atherosclerosis was significantly smaller at 8 weeks after injury than 12 weeks after injury $\left(174.7 \pm 35.0^{\circ}\right.$ versus $245.0 \pm$ $\left.45.1^{\circ} ; P=0.01\right)$ in the 6 rabbits that underwent repeat OCT. The site within the lumen that contained the plaque bulged into the adventitia rather than toward the lumen (Figure $4(\mathrm{e}))$.

3.4. IVUS Findings. At eight weeks after injury, eccentric plaques with hypoechoic regions were observed in the IVUS images of right CCAs of all rabbits (Figure 5(a)), whereas no obvious atherosclerotic plaques were observed in the left CCAs (Figure 5(b)). At twelve weeks after injury, plaque size was increased in the right CCAs of the 6 rabbits that underwent repeat IVUS (from $2.02 \pm 0.59$ to $3.69 \pm 1.22 \mathrm{~mm}^{2} ; P=$ 0.01 ). Marked progression was clearly observed in the same 


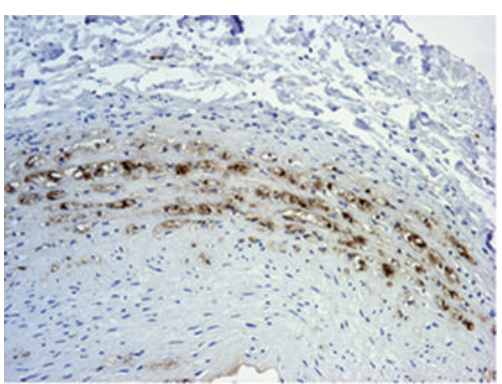

(a)

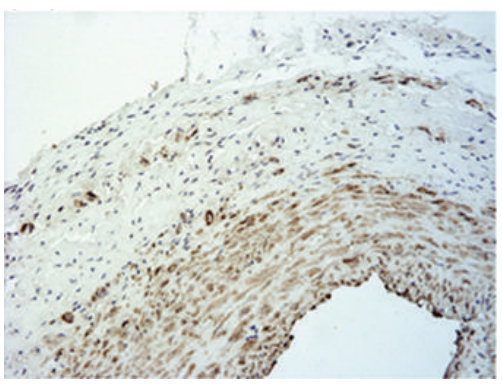

(c)

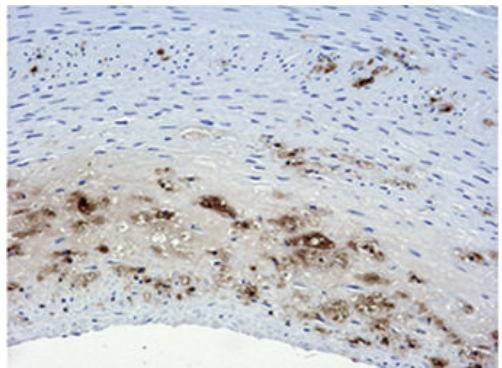

(b)

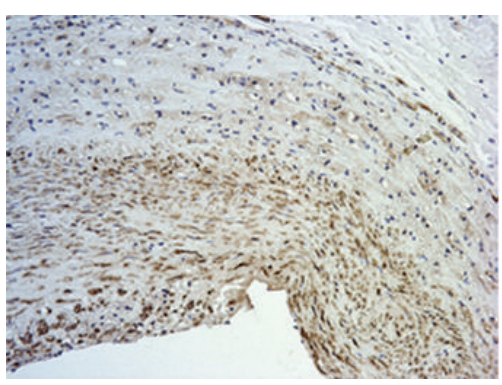

(d)

Figure 3: Immunohistochemical stains for macrophages ((a) and (b)) and smooth muscle cells ((c) and (d)). Original magnification, $\times 200$.

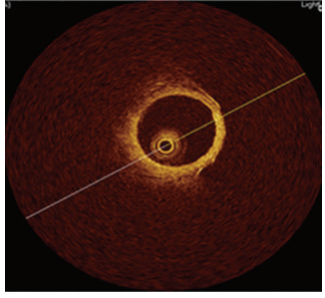

(a)

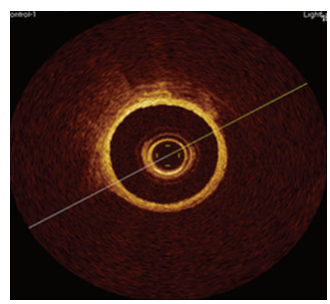

(c)

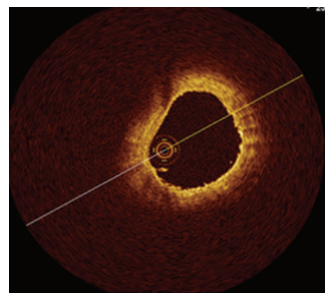

(b)

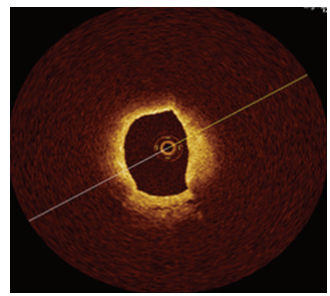

(d)

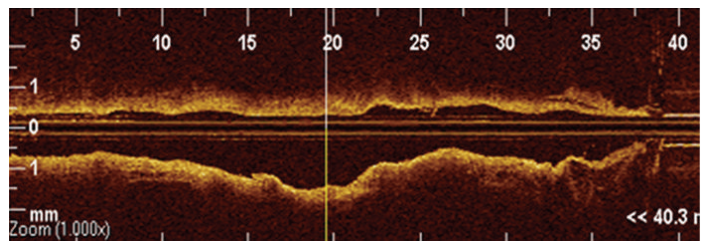

(e)

Figure 4: Representative optical coherence tomography images. At 24 hours after injury, no thrombus is detected in the lumen (a). At 8 weeks after injury, atherosclerosis of the right common carotid arteries is evident (b), but not in the control (c). (d) is the same cross-section as (b) and shows evident plaque growth at 12 weeks. In the same rabbit, a typical arterial enlargement is seen (e). plaque 12 weeks after injury (Figure 5(c)). At the site with atherosclerosis, evident vessel enlargement of right CCA is seen in Figure 5(d), indicating prominent positive remodeling. At eight weeks after jury, six atherosclerotic lesions $(50 \%)$ with significant positive remodeling were observed (RI: $1.19 \pm 0.07)$. at twelve weeks after injury, remodeling index was increased in the right CCAs of the 6 rabbits that underwent repeat IVUS (from $1.01 \pm 0.12$ to $1.09 \pm 0.15$; $P=0.02)$.

\section{Discussion}

We successfully developed a novel, inexpensive, and reproducible animal model of atherosclerotic plaques. This model showed progressive atherosclerotic changes from injured segments induced by $\mathrm{FeCl}_{3}$ to adjacent noninjured arterial segments and macrophage migration from the media to the neointima, finally forming eccentric atherosclerotic lesions with positive remodeling. This model also permitted serial evaluation of atherosclerosis progression in the same rabbit using two imaging modalities with a high success rate and low mortality. Serial investigation showed that the remodeling index and eccentricity became bigger with plaque growth over time.

Numerous models of atherosclerosis have been reported in rabbits $[1,10]$. These range from mechanical injury to electric injury as well as gene knockouts disclose the mechanisms underlying the atherosclerotic process and develop new antiatherosclerotic strategies $[10,11]$. A high concentration of $\mathrm{FeCl}_{3}$ was applied to produce animal models of arterial thrombosis for study of novel antithrombotic 


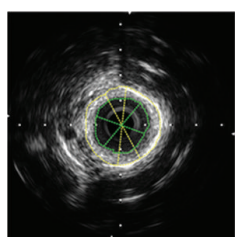

(a)

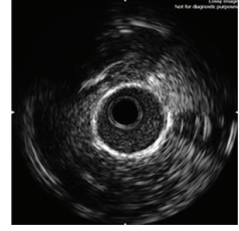

(b)

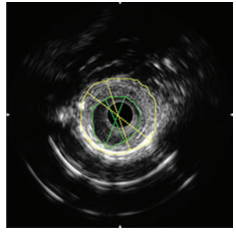

(c)

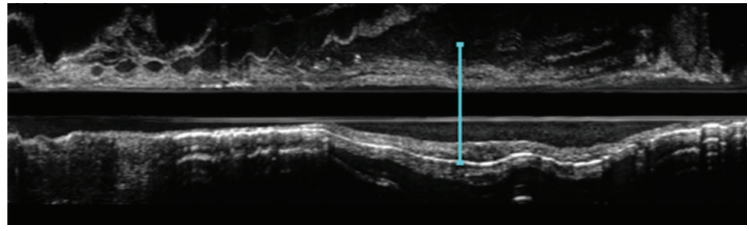

(d)

FIGURE 5: Representative intravascular ultrasound images. An eccentric plaque is observed in right common carotid arteries 8 weeks (a) and 12 weeks (c) after injury, but not in the control (b). Marked enlargement of the vessel wall at the site of atherosclerosis is clearly observed (e).

agents [3, 12]. Our previous animal study demonstrated the feasibility of OCT for the detection of acute thrombosis in vivo [7]. In the present study, a favorable $\mathrm{FeCl}_{3}$ concentration and injury time was established by a preliminary study as assessed by in vivo serial OCT investigation and histological detection. This was done to induce moderate injury to the arterial wall and to avoid causing an evident thrombus. Three rabbits were chosen randomly and underwent OCT $24 \mathrm{~h}$ after $\mathrm{FeCl}_{3}$-induced injury: no thrombus was seen. We confirmed that a combination of $\mathrm{FeCl}_{3}$-induced chemical injury (by surgical means) and a high-cholesterol diet could induce the formation of atherosclerotic plaques. Similar to other models [13], the plaque of this model was rich in macrophages. Macrophages have been implicated in every stage of coronary atherosclerosis from its initiation representation to clinical its presentation.

One feature of the plaques in this model was their eccentric nature and that they did not cause severe luminal stenosis in the CCAs 12 weeks after injury. One possible reason for this finding is that the region of injury to the vessel wall was local. This feature makes some invasive imaging methods (e.g., IVUS and OCT) feasible for imaging carotid arteries with atherosclerosis in rabbits. Some animal studies have shown that a conventional model of atherosclerosis using a combination of balloon injury and an atherogenic diet can cause severe lumen stenosis (possibly due to balloon-induced diffuse injury to the intima). Eccentric stenosis was independently related to recent cerebrovascular events [14].

Another feature of this model was that the arteries underwent expansive remodeling in response to plaque growth over time. One possible explanation for this feature was death of cells in vessel wall due to $\mathrm{FeCl}_{3}$-induced injury as well as a decrease in the retractility of the vessel wall. This allows the plaque to grow outward rather than inward towards the lumen. After the first description by Glagov and colleagues [15], this process of compensatory arterial enlargement has been widely studied and has demonstrated that it is a crucial marker of plaque vulnerability [16]. Many studies on humans have shown that the arterial wall can remodel itself by increasing its vessel diameter to accommodate the plaque without narrowing the lumen [17]. There is growing evidence that expansive remodeling can contribute to plaque vulnerability [18]. Recent clinical studies have indicated that outward remodeling may be associated with an increased risk of cardiovascular events. The characteristics that positive remodeling situation became more severe over time in this model, are beneficial for developing new method strategies of antiremodeling.

In the clinical setting, IVUS and OCT have been used to study the size and composition of plaques and to evaluate intervention strategies [19-21]. Plaque burden and the remodeling index can be obtained from IVUS offline analyses even in the presence of large plaques. OCT is analogous to ultrasound except that it is uses light instead of sound $[8,20]$. OCT offers considerable resolution and has high sensitivity and specificity for distinct plaque components but has low penetration [22]. In animal studies using the same surrogates applied in clinical settings, it is of better suitability for translation to humans. In the present study, we evaluated the development of a novel model of atherosclerosis by combining IVUS and OCT. We demonstrated the feasibility of IVUS and OCT to evaluate the same artery twice. It is very useful for clinician to evaluate the consistency between the parameters, reflecting the same characteristics of atherosclerosis, from different imaging modalities and to investigate the relationship between the parameters, reflecting the different characteristics of atherosclerosis. Compared with the balloon-injury model [23], creation of our model was relatively simple and did not necessitate dissection of the main branch of the carotid artery. This feature may be associated with a lower mortality when undertaking multiple invasive imaging in the same artery of rabbits. A combination of imaging modalities is useful to obtain more comprehensive information of atherosclerosis progression, to improve our understanding of atherosclerosis, and to evaluate the effect of intervention strategies.

One of the major drawbacks of this model is that no spontaneous intraplaque hemorrhage, plaque rupture or thrombus was observed. Plaque disruption and ensuing thrombus are the direct cause of cardiovascular and cerebrovascular acute events. Another limitation of this study was that our present study was based on a small sample size; however, a greater number of animals were not ethically justifiable. A longer followup study with a larger sample is needed to obtain more parameters of this model.

\section{Conclusions}

We demonstrated, for the first time, a novel model of atherosclerosis induced by $\mathrm{FeCl}_{3}$. The model is simple, fast, inexpensive, and reproducible and has a high success rate. The eccentric plaques and remodeling of plaques were 
common in this model. We successfully carried out IVUS and OCT examinations twice in the same lesion within a relatively long period of time. We believe that this model may be a good method for studying the physiology, pathology, and of the therapeutic effects of various agents over time.

\section{Acknowledgments}

B. Yu was supported by Grant no. 30871064 from the National Natural Science Foundation of China. The authors would like to thank Xia $\mathrm{Gu}$ for her excellent help in editing and Songyu Tian for his help in model making. They also thank International Science Editing for revision of the paper. They declare no conflict of interests.

\section{References}

[1] W. C. Dornas, T. T. Oliveira, L. E. Augusto, and T. J. Nagem, "Experimental atherosclerosis in rabbits," Arquivos Brasileiros de Cardiologia, vol. 95, no. 2, pp. 272-278, 2010.

[2] X. Xian, Y. Ding, L. Zhang et al., "Enhanced atherothrombotic formation after oxidative injury by $\mathrm{FeCl}_{3}$ to the common carotid artery in severe combined hyperlipidemic mice," Biochemical and Biophysical Research Communications, vol. 385, no. 4, pp. 563-569, 2009.

[3] D. J. Evans, L. E. Jackman, J. Chamberlain et al., "Platelet $\mathrm{P} 2 \mathrm{Y}_{12}$ receptor influences the vessel wall response to arterial injury and thrombosis," Circulation, vol. 119, no. 1, pp. 116-122, 2009.

[4] E. Regar, "Invasive imaging technologies: can we reconcile light and sound?" Journal of Cardiovascular Medicine, vol. 12, no. 8, pp. 562-570, 2011.

[5] H. Yabushita, B. E. Bouma, S. L. Houser et al., "Characterization of human atherosclerosis by optical coherence tomography," Circulation, vol. 106, no. 13, pp. 1640-1645, 2002.

[6] M. Pynn, K. Schafer, S. Konstantinides, and M. Halle, "Exercise training reduces neointimal growth and stabilizes vascular lesions developing after Injury in apolipoprotein e-deficient mice," Circulation, vol. 109, no. 3, pp. 386-392, 2004.

[7] L. Meng, B. Lv, S. Zhang, and B. Yv, "In vivo optical coherence tomography of experimental thrombosis in a rabbit carotid model," Heart, vol. 94, no. 6, pp. 777-780, 2008.

[8] M. Zimarino, F. Prati, E. Stabile et al., "Optical coherence tomography accurately identifies intermediate atherosclerotic lesions-an In vivo evaluation in the rabbit carotid artery," Atherosclerosis, vol. 193, no. 1, pp. 94-101, 2007.

[9] M. Cilingiroglu, J. H. Oh, B. Sugunan et al., "Detection of vulnerable plaque in a murine model of atherosclerosis with optical coherence tomography," Catheterization and Cardiovascular Interventions, vol. 67, no. 6, pp. 915-923, 2006.

[10] G. Vilahur, T. Padro, and L. Badimon, "Atherosclerosis and thrombosis: insights from large animal models," Journal of Biomedicine and Biotechnology, vol. 2011, Article ID 907575, 12 pages, 2011.

[11] R. Kamimura, S. Suzuki, H. Sakamoto, N. Miura, K. Misumi, and K. Miyahara, "Development of atherosclerotic lesions in cholesterol-loaded rabbits," Experimental Animals, vol. 48, no. 1, pp. 1-7, 1999.

[12] S. J. Korporaal, I. Meurs, A. D. Hauer et al., "Deletion of the high-density lipoprotein receptor scavenger receptor BI in mice modulates thrombosis susceptibility and indirectly affects platelet function by elevation of plasma free cholesterol," Arteriosclerosis, Thrombosis, and Vascular Biology, vol. 31, no. 1, pp. 34-42, 2011.

[13] T. M. Bocan, S. B. Mueller, P. D. Uhlendorf, E. Ferguson, and R. S. Newton, "Dietary and mechanically induced rabbit iliacfemoral atherosclerotic lesions: a chemical and morphologic evaluation," Experimental and Molecular Pathology, vol. 54, no. 3, pp. 201-217, 1991.

[14] T. Ohara, K. Toyoda, R. Otsubo et al., "Eccentric stenosis of the carotid artery associated with ipsilateral cerebrovascular events," American Journal of Neuroradiology, vol. 29, no. 6, pp. 1200-1203, 2008.

[15] S. Glagov, E. Weisenberg, C. K. Zarins, R. Stankunavicius, and G. J. Kolettis, "Compensatory enlargement of human atherosclerotic coronary arteries," The New England Journal of Medicine, vol. 316, no. 22, pp. 1371-1375, 1987.

[16] I. Sipahi, E. M. Tuzcu, P. Schoenhagen et al., "Compensatory enlargement of human coronary arteries during progression of atherosclerosis is unrelated to atheroma burden: serial intravascular ultrasound observations from the REVERSAL trial," European Heart Journal, vol. 27, no. 14, pp. 1664-1670, 2006.

[17] A. M. Varnava, P. G. Mills, and M. J. Davies, "Relationship between coronary artery remodeling and plaque vulnerability," Circulation, vol. 105, no. 8, pp. 939-943, 2002.

[18] O. C. Raffel, F. M. Merchant, G. J. Tearney et al., "In vivo association between positive coronary artery remodelling and coronary plaque characteristics assessed by intravascular optical coherence tomography," European Heart Journal, vol. 29, no. 14, pp. 1721-1728, 2008.

[19] J. C. Tardif, F. Lesage, F. Harel, P. Romeo, and J. Pressacco, "Imaging biomarkers in atherosclerosis trials," Circulation, vol. 4, no. 3, pp. 319-333, 2011.

[20] D. Nemirovsky, "Imaging of high-risk plaque," Cardiology, vol. 100, no. 4, pp. 160-175, 2003.

[21] L. Cho, B. E. Lewis, L. H. Steen, and F. S. Leya, "Thiazolidinediones do not reduce target vessel revascularization in diabetic patients undergoing percutaneous coronary intervention," Cardiology, vol. 104, no. 2, pp. 97-100, 2005.

[22] E. Regar, J. Ligthart, N. Bruining, and G. V. Soest, "The diagnostic value of intracoronary optical coherence tomography," Herz, pp. 417-429, 2011.

[23] A. E. Yanni, "The laboratory rabbit: an animal model of atherosclerosis research," Laboratory Animals, vol. 38, no. 3, pp. 246-256, 2004. 


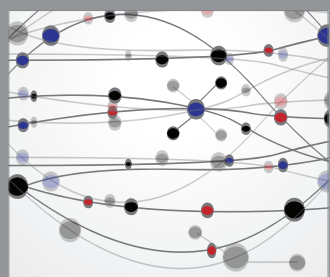

The Scientific World Journal
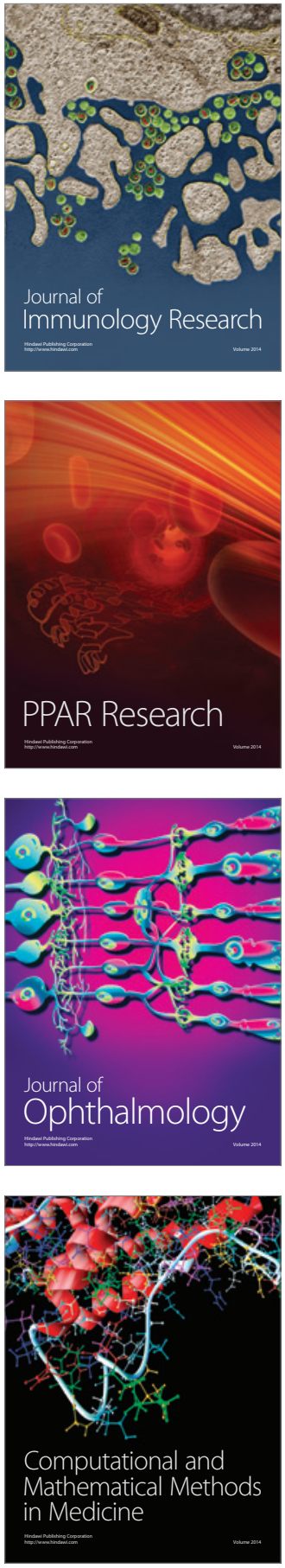

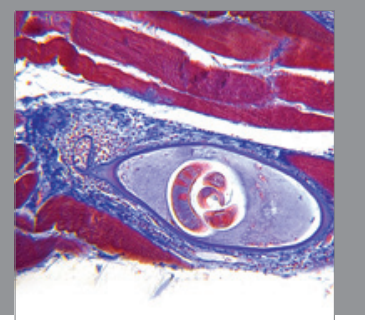

Gastroenterology

Research and Practice
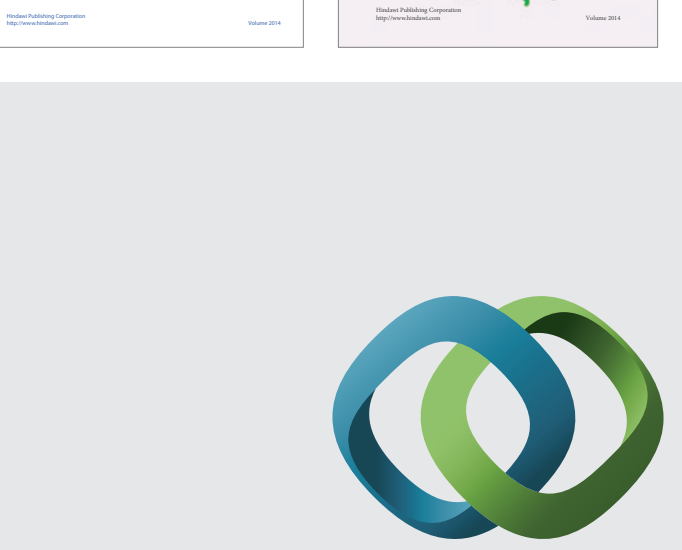

\section{Hindawi}

Submit your manuscripts at

http://www.hindawi.com
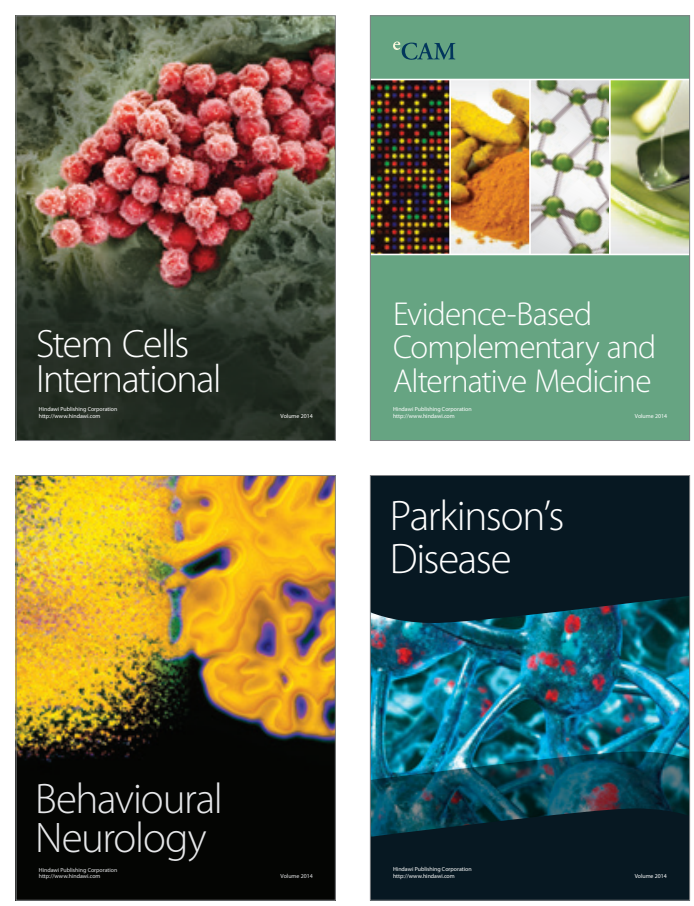

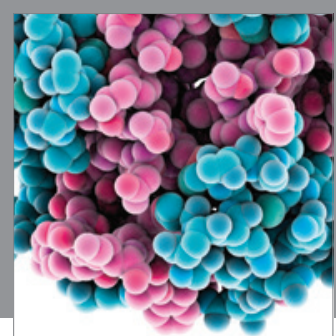

Journal of
Diabetes Research

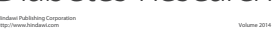

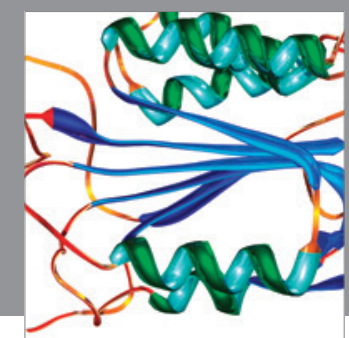

Disease Markers
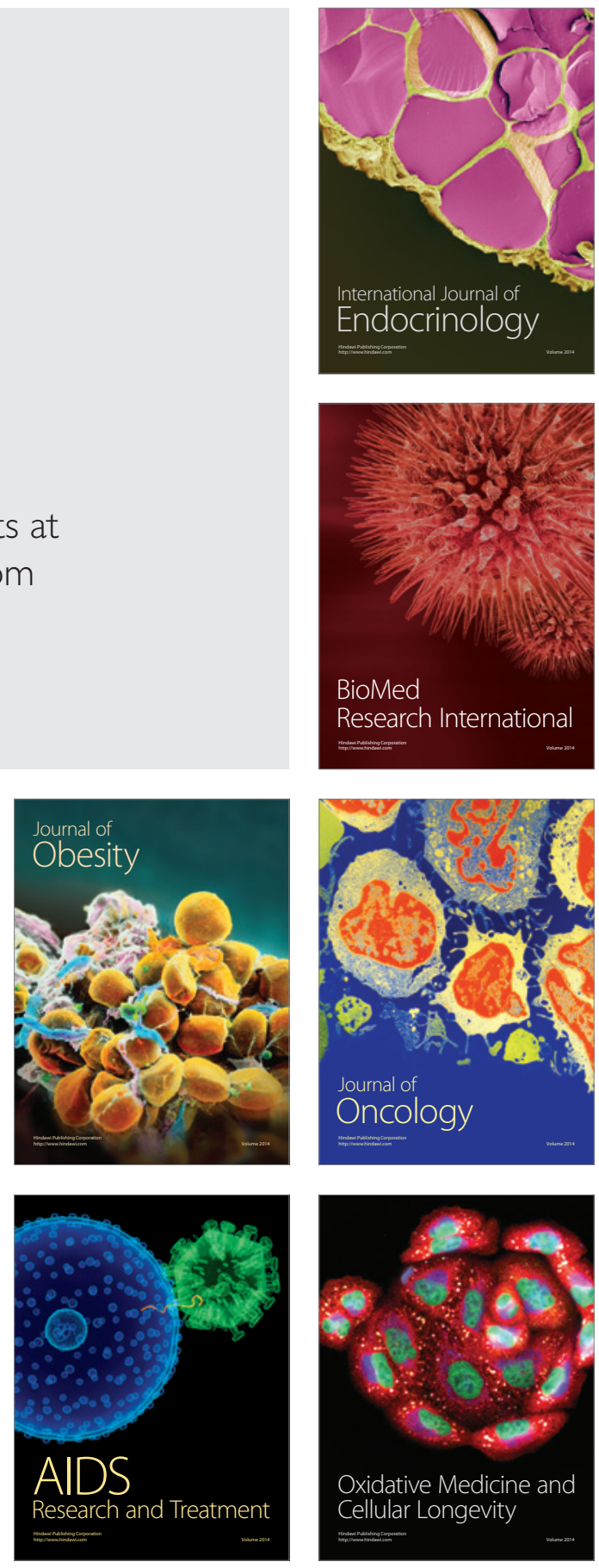